\title{
EDITORIAL
}

\section{Interstitial lung disease: new challenges and evolving phenotypes}

\author{
V. Cottin
}

$\mathbf{T}$ here have been major changes in the field of interstitial lung disease (ILD) in the past decade, mostly in disease classification and diagnostic processes, and in the role of high-resolution computed tomography (HRCT) of the chest in assessment of diagnosis and prognosis. In the present issue of the European Respiratory Review, HARARI and CAMINATI [1] review the current evidence related to ILD. This editorial emphasises some of the most important challenges in idiopathic pulmonary fibrosis (IPF) as well as some of the recent and changing concepts among the many phenotypes of ILD.

\section{CURRENT CHALLENGES IN IPF}

IPF is the most severe of the chronic ILDs. One major advance has been made by the better delineating of the disease and the well-adopted international definition of IPF that allowed large clinical trials to be conducted. As some inconsistencies remain in the classification of the idiopathic interstitial pneumonias, an international collaborative task force has begun working under the auspices of the American Thoracic Society and the European Respiratory Society on a possible revision of the current classification. Among many other issues, the task force will have to address the still ongoing debate of usual interstitial pneumonia versus nonspecific pneumonia and their frequently overlapping features; the recently individualised syndrome of combined pulmonary fibrosis and emphysema [2]; and new histopathological patterns that might correspond to clinically relevant entities (e.g. acute fibrinous and organising pneumonia, smoking-related interstitial fibrosis [3], etc.) [4]; and will question the adequacy of maintaining, in the group of idiopathic interstitial pneumonias, entities that are mostly due to environmental exposure or tobacco smoking and therefore not idiopathic (e.g. desquamative interstitial pneumonia) or that are characterised by prominent alveolar, rather than interstitial, changes in histopathology (e.g. organising pneumonia).

Several messages emerge from the review by HARARI and CAMINATI [1] regarding the epidemiology of IPF. First, the incidence, prevalence, and specific mortality of IPF seem to have increased in recent years [5]. Secondly, much remains to be learned about the relative contribution of tobacco smoking,

CORRESPONDENCE: Hospices Civils de Lyon, Louis Pradel Hospital, National Reference Centre for Rare Pulmonary Diseases, Dept of Respiratory Diseases, Lyon I University, UMR 754, Lyon, F-69677, France,.E-mail: vincent.cottin@chu-lyon.fr

Received: April 07 2010; Accepted: April 072010

PROVENANCE: Submitted article, peer reviewed. genetic predisposition, cardiovascular disease or risk factors, and IPF. For example, the relative risk of developing acute coronary syndrome or deep vein thrombosis is increased in IPF [6], as may be the risk of obstructive sleep apnoea [7].

Pulmonary physicians will also remember practical messages, such as the importance of considering the age of the patient, in the diagnostic approach of ILD. While the median age of IPF may be frequently underestimated (the mean age is $\sim 65$ yrs in large clinical trials or series [8], yet the peak incidence of IPF occurs between 75 and 85 yrs of age in epidemiological studies [5]). Bronchoalveolar lavage only modestly contributes to the differential diagnosis between IPF and nonspecific interstitial pneumonia $[9,10]$, but its importance in the process of diagnosing ILDs at large is well established.

Updated guidelines on the management of IPF have been presented at the 2009 Annual Congress of the European Respiratory Society. Although no therapy has yet proven clinically useful in this devastating disease, ongoing and future trials carry significant hope. Controlling the progression of IPF may require the use of combined therapy, as in other areas, including cancer [11], infection with HIV or tuberculosis. It is hoped that future progress made in the drug therapy of IPF will also impact the management of many other chronic diseases that encompass progressive fibrosis or that involve similar processes, such as deregulation of myofibroblast recruitment or cell proliferation.

Two main challenges in IPF are related to situations that significantly contribute to the mortality of this devastating disease, namely acute exacerbations of disease [12] and severe pulmonary hypertension [13]. The former is defined by the rapid deterioration of IPF during the course of disease that cannot be attributed to an identified cause. The recent international consensus statement [14] will undoubtedly boost research developments regarding pathophysiology and management of this severe event. While many groups empirically use corticosteroids and often immunosuppressive therapy, this approach is not yet supported by evidence, and clinical research is eagerly needed. Other conditions characterised by rapid progression of disease but not fulfilling the definition of acute exacerbations, coined accelerated disease or subacute exacerbation of IPF, also need further evaluation.

Pulmonary hypertension confirmed by right heart catheterisation is present in more than one-third of patients with IPF referred for lung transplantation, and significantly affects 
mortality, with a 1-yr mortality of $28 \%$ in patients with pulmonary hypertension compared with $5 \%$ in its absence [15]. Whether specific therapy of pulmonary hypertension may impact the outcome is currently investigated in observational studies and clinical trials.

Although it has not translated into effective therapy, much progress has been made in the understanding of fibrogenesis in recent years. The various origins of fibroblasts in the fibrotic lung (resident lung fibroblasts, epithelial lung cells through epithelial mesenchymal differentiation and circulating progenitors known as fibrocytes, which might be used as biomarkers of active fibrosis in the future) is the subject of active research, as this may give new insights into novel therapies. Genomic studies have showed that the gene expression profile of IPF differs from that of diseases where significant inflammation is found, such as hypersensitivity pneumonitis [16]. Many of the genes with increased expression in IPF encode proteins associated with extracellular matrix formation, cell proliferation, migration, invasion and cell morphology, and proteins expressed in smooth muscle cells [17], reminiscent of that observed in cancer biology [11]. This observation holds true in studies on lungs obtained from patients with the accelerated variant of IPF [18] or acute exacerbation of IPF [19]. It is not clear whether activation of inflammation pathways is not key to the pathogenesis of IPF, or if published genomic studies reflected prominent remodelling in the fibrotic lung while failing to catch more subtle deregulation of other pathways involved in the interplay between injured pneumocytes and myofibroblasts.

\section{EVOLVING PHENOTYPES OF ILD}

Several phenotypes of ILD are being increasingly recognised that progressively modify the clinical approach and patients' care.

The entity of combined pulmonary fibrosis and emphysema has been classified in the group of smoking-induced chronic lung disease $[2,20]$. It is characterised by the association of distinct features, including tobacco smoking, severe dyspnoea, unexpected subnormal spirometry, severely impaired transfer capacity for carbon monoxide, hypoxaemia on exercise and characteristic imaging features. Although the pathophysiology of this condition is not well understood, the causative role of tobacco smoking is obvious. Diagnosing this syndrome has clinical relevance, because it carries an increased risk of developing pulmonary hypertension with increased mortality [21, 22].

ILD associated with connective tissue disease is a complex field [23] that can be viewed as a model of pulmonary fibrosis with a known aetiological context. Survival in patients with ILD associated with connective tissue disease is better than that of IPF [24]. Studies concerned with the determinants of long-term prognosis are emerging [25]; for example, the pattern on HRCT or the extent of ILD on HRCT are predictive of survival in patients with systemic sclerosis or rheumatoid arthritis, respectively [26, 27]. Differentiating IPF from occult or undifferentiated connective tissue disease is a recurring question in clinical care, with frequent therapeutic consequences, especially in patients with a histological pattern of nonspecific interstitial pneumonia. "Idiopathic" nonspecific interstitial pneumonia may thus in some cases correspond to formes frustes of connective tissue disease limited to the lung [28], hence the recently proposed terminology of "lung-dominant connective tissue disease" to qualify this condition [29]. Furthermore, the ILD may be the presenting manifestation of the connective tissue disease in a patient with yet-to-come extrarespiratory manifestations; this has been described most widely in patients with nonspecific interstitial pneumonia, but also in those with organising pneumonia, as reported in this issue of the European Respiratory Review [30].

The role of auto-antibodies needs clarification. Although circulating auto-antibodies are frequently found in patients with interstitial pneumonia and subtle or no extra-thoracic manifestations, the survival of patients with IPF is not altered by the presence of auto-antibodies [31]. Several studies have reported the presence of anti-neutrophil cytoplasmic antibodies in patients with pulmonary fibrosis; however, the clinical relevance of this observation is incompletely known. Systemic vasculitis and, particularly, microscopic polyangiitis have been observed during the follow-up of patients with IPF who had anti-neutrophil cytoplasmic antibodies [32].

Many other recent developments in ILD that cannot be mentioned here are analysed in depth in the review article by HARARI and CAMINATI [1]. For example, the first international guidelines on lymphangioleiomyomatosis [33] cover all aspects of diagnostic and therapeutic management of this orphan disease and will undoubtedly facilitate multicentre clinical trials and stimulate collaborative studies, as it has been the case in IPF.

\section{STATEMENT OF INTEREST}

V. Cottin has participated as a member of a steering committee, member of a data monitoring committee, member of an adjudication committee and investigator in clinical trials sponsored by Actelion, Boehringer, Centocor, Intermune and Wyeth, and has been a lecturer for Actelion.

\section{REFERENCES}

1 Harari S, Caminati A. Update on diffuse parenchymal lung disease. Eur Respir Rev 2010; 19: 97-108.

2 Cottin V, Nunes H, Brillet PY, et al. Combined pulmonary fibrosis and emphysema: a distinct underrecognised entity. Eur Respir $J$ 2005; 26: 586-593.

3 Katzenstein AL, Mukhopadhyay S, Zanardi C, et al. Clinically occult interstitial fibrosis in smokers: classification and significance of a surprisingly common finding in lobectomy specimens. Hum Pathol 2010; 41: 316-325.

$4 \mathrm{du}$ Bois R, King TE Jr. Challenges in pulmonary fibrosis $\times 5$ : the NSIP/UIP debate. Thorax 2007; 62: 1008-1012.

5 Gribbin J, Hubbard RB, Le Jeune I, et al. Incidence and mortality of idiopathic pulmonary fibrosis and sarcoidosis in the UK. Thorax 2006; 61: 980-985.

6 Hubbard RB, Smith C, Le Jeune I, et al. The association between idiopathic pulmonary fibrosis and vascular disease: a populationbased study. Am J Respir Crit Care Med 2008; 178: 1257-1261.

7 Lancaster LH, Mason WR, Parnell JA, et al. Obstructive sleep apnea is common in idiopathic pulmonary fibrosis. Chest 2009; 136: 772-778.

8 King TE Jr, Behr J, Brown KK, et al. BUILD-1: a randomized placebo-controlled trial of bosentan in idiopathic pulmonary fibrosis. Am J Respir Crit Care Med 2008; 177: 75-81. 
9 Veeraraghavan S, Latsi PI, Wells AU, et al. BAL findings in idiopathic nonspecific interstitial pneumonia and usual interstitial pneumonia. Eur Respir J 2003; 22: 239-244.

10 Ohshimo S, Bonella F, Cui A, et al. Significance of bronchoalveolar lavage for the diagnosis of idiopathic pulmonary fibrosis. Am J Respir Crit Care Med 2009; 179: 1043-1047.

11 Vancheri C, Failla M, Crimi N, et al. Idiopathic pulmonary fibrosis: a disease with similarities and links to cancer biology. Eur Respir J 2010; 35: 496-504.

12 Daniels CE, Yi ES, Ryu JH. Autopsy findings in 42 consecutive patients with idiopathic pulmonary fibrosis. Eur Respir J 2008; 32: 170-174.

13 Behr J, Ryu JH. Pulmonary hypertension in interstitial lung disease. Eur Respir J 2008; 31: 1357-1367.

14 Collard HR, Moore BB, Flaherty KR, et al. Acute exacerbations of idiopathic pulmonary fibrosis. Am J Respir Crit Care Med 2007; 176: 636-643.

15 Lettieri CJ, Nathan SD, Barnett SD, et al. Prevalence and outcomes of pulmonary arterial hypertension in advanced idiopathic pulmonary fibrosis. Chest 2006; 129: 746-752.

16 Selman M, Pardo A, Barrera L, et al. Gene expression profiles distinguish idiopathic pulmonary fibrosis from hypersensitivity pneumonitis. Am J Respir Crit Care Med 2006; 173: 188-198.

17 Boon K, Bailey NW, Yang J, et al. Molecular phenotypes distinguish patients with relatively stable from progressive idiopathic pulmonary fibrosis (IPF). PLoS One 2009; 4: e5134.

18 Selman M, Carrillo G, Estrada A, et al. Accelerated variant of idiopathic pulmonary fibrosis: clinical behavior and gene expression pattern. PLoS One 2007; 2: e482.

19 Konishi K, Gibson KF, Lindell KO, et al. Gene expression profiles of acute exacerbations of idiopathic pulmonary fibrosis. Am J Respir Crit Care Med 2009; 180: 167-175.

20 Balbi B, Cottin V, Singh S, et al. Smoking-related lung diseases: a clinical perspective. Eur Respir J 2010; 35: 231-233.

21 Mejia M, Carrillo G, Rojas-Serrano J, et al. Idiopathic pulmonary fibrosis and emphysema: decreased survival associated with severe pulmonary arterial hypertension. Chest 2009; 136: 10-15.
22 Cottin V, Le Pavec J, Prevot G, et al. Pulmonary hypertension in patients with combined pulmonary fibrosis and emphysema syndrome. Eur Respir J 2010; 35: 105-111.

23 Antoniou KM, Margaritopoulos G, Economidou F, et al. Pivotal clinical dilemmas in collagen vascular diseases associated with interstitial lung involvement. Eur Respir J 2009; 33: 882-896.

24 Park JH, Kim DS, Park IN, et al. Prognosis of fibrotic interstitial pneumonia: idiopathic versus collagen vascular disease-related subtypes. Am J Respir Crit Care Med 2007; 175: 705-711.

25 Gilson M, Zerkak D, Wipff J, et al. Prognostic factors for lung function in systemic sclerosis: prospective study of 105 cases. Eur Respir J 2010; 35: 112-117.

26 Kim EJ, Elicker BM, Maldonado F, et al. Usual interstitial pneumonia in rheumatoid arthritis-associated interstitial lung disease. Eur Respir J 2010; 35: 1322-1328.

27 Goh NS, Desai SR, Veeraraghavan S, et al. Interstitial lung disease in systemic sclerosis: a simple staging system. Am J Respir Crit Care Med 2008; 177: 1248-1254.

28 Kinder BW, Collard HR, Koth L, et al. Idiopathic nonspecific interstitial pneumonia: lung manifestation of undifferentiated connective tissue disease? Am J Respir Crit Care Med 2007; 176: 691-697.

29 Fischer A, West SG, Swigris JJ, et al. Connective tissue diseaseassociated interstitial lung disease: a call for clarification. Chest 2010; (in press).

30 Henriet AC, Diot E, Marchand-Adam S, et al. Organising pneumonia can be the inaugural manifestation in connective tissue diseases, including Sjögren's syndrome. Eur Respir Rev 2010; 19: 161-163.

31 Song JW, Do KH, Kim MY, et al. Pathologic and radiologic differences between idiopathic and collagen vascular diseaserelated usual interstitial pneumonia. Chest 2009; 136: 23-30.

32 Foulon G, Delaval P, Valeyre D, et al. ANCA-associated lung fibrosis: analysis of 17 patients. Respir Med 2008; 102: 1392-1398.

33 Johnson SR, Cordier JF, Lazor R, et al. European Respiratory Society guidelines for the diagnosis and management of lymphangioleiomyomatosis. Eur Respir J 2010; 35: 14-26. 